\title{
Pediatric Gram-Negative Bacteremia: Hidden Agenda
}

\author{
Elham Essa Bukhari ${ }^{1} \quad$ Abdulkarim A. Alrabiaah ${ }^{1}$ \\ ${ }^{1}$ Department of Pediatric Infectious Disease, College of Medicine, \\ King Saud University Medical City and King Saud University, \\ Riyadh, Saudi Arabia

\begin{abstract}
Address for correspondence Elham Essa Bukhari, MD, Department of Pediatric Infectious Disease, Faculty of Medicine, King Saud University Medical City and King Saud University, P.O. Box 2925, Riyadh 11461,
\end{abstract} \\ Saudi Arabia (e-mail: ebukhari@ksu.edu.sa).
}

J Child Sci 2018;8:e1-e6.

\begin{abstract}
Keywords

- bacteremia

- community-acquired

- healthcare-associated

- hospital-acquired

Background Recently, new types of community-onset bacteremia have been introduced as healthcare associated (HCA) in which the infection onset started outside the hospital and there were interactions with the healthcare system. Little data exist differentiating community-acquired (CA) and HCA bacteremia from hospital-acquired bacteremia (HA).

Objectives This article determines differences in the epidemiological characteristics and bacteriology of community-onset (i.e., CA and HCA) and HA gram-negative bacteremia in Saudi pediatric patients.

Methods We conducted a prospective cohort of all pediatric patients diagnosed with gram-negative bacteremia at the King Khalid University Hospital over a year (2015). We received daily electronic notifications of all blood culture positive cases for gramnegative bacilli.

Results A total of 92 children were hospitalized with gram-negative bacteremia; among these $64(71.1 \%)$ were with HA bacteremia, 20 (21.1\%) with CA bacteremia, and $8(7.8 \%)$ with HCA bacteremia. Urinary tract infection was common clinical presentation (50\%) in the patients diagnosed with CA and HCA bacteremia. Up to $92 \%$ of HA bacteremia and $2 \%$ of CA bacteremia were presented with septic shock. The most common gram-negative bacteria causing bacteremia is Klebsiella pneumoniae, constituting almost $29.3 \%$ of all organisms, and was only isolated from HA bacteremia. The antimicrobial susceptibility among the 92 isolates showed that the organisms were nonextended spectrum $\beta$-lactamase (non-ESBL) in $90 \%$, and $10 \%$ of the isolates were ESBL. There was a significant difference in the total frequency of isolates between $C A$ and HA incidences, regardless of ESBL or non-ESBL $(p<0.001)$.

Conclusion The most common type of gram-negative bacteremia is HA bacteremia followed by the CA and HCA bacteremia.
\end{abstract}

\section{Introduction}

Bacteremia is a major pediatric health care problem. Despite great developments in therapy and supportive care, bacteremia still represents a major cause of morbidity and mortality. ${ }^{1,2}$ In particular, gram-negative bacteremia has increasingly been reported among the pediatric population. ${ }^{3}$ Recently, community-onset bacteremia was reclassified, due to increasing numbers of clinical outpatient treatments, into healthcare-associated (HCA) and community-acquired (CA) bacteremia. ${ }^{4-8}$ Previous studies that made a comparison between hospital-acquired nosocomial (HA) gram-negative received

September 30, 2017 accepted after revision November 30, 2017
DOI https://doi.org/

10.1055/s-0037-1618564. ISSN 2474-5871.
Copyright (c) 2018 Georg Thieme Verlag License terms KG Stuttgart · New York 
bacteremia and CA bacteremia found that there was an increased rate of bacteremia due to drug-resistant pathogens. $^{9-11}$ Studying the varying microbiological patterns of bacteremia in children, gram-negative septicemia was categorized in two studies to be up to 71.87 and $73 \%$, respectively, of the culture-positive cases. ${ }^{12,13}$ Furthermore, recently, there has been an increase in the number of HCA gramnegative bacteremia among hospitalized patients with high rate of multidrug-resistant (MRO) bacteremia. ${ }^{14,15}$ The objective of this study was to analyze the differences between the three types of gram-negative bacteremia with regard to the comorbidities, clinical characteristics, etiology, and outcomes among Saudi children in the King Khalid University Hospital.

\section{Methods}

\section{Study Design}

This study was conducted at the King Khalid University Hospital, a 1,100-bed hospital with 135 monthly admissions in pediatric wards. It is a major teaching hospital in Riyadh, Saudi Arabia, providing both primary and tertiary medical care. The study was conducted between January 11, 2015, and December 27, 2015. Data were purposely collected from pediatric patients below 15 years of age with gram-negative blood infections, using daily computerized laboratory data and medical record information system. Standardized data forms were used to record demographic details, including underlying diseases, hospital unit, and exposure to the healthcare system in the previous years.

\section{Statistical Analysis}

All data were collected in an Excel sheet and were analyzed using the analysis of variance (ANOVA) method for finding differences among the three types of bacteremia. Results were considered significant if $p<0.05$. Ethical approval for the study was obtained from the ethics committee of the hospital.

\section{Definitions}

Gram-negative bacteremia is defined as the isolation of gram-negative bacilli in a blood culture specimen. Clinically, significant bacteremia is defined as at least one positive blood culture, together with clinical features compatible with systemic inflammatory response syndrome. Patients were diagnosed with CA, gram-negative bacteremia if their first positive blood culture results were obtained from blood samples drawn within 48 hours after hospital admission. Cases of HCA bacteremia were diagnosed if one or more of the following criteria was fulfilled: outpatient treatment (patient treated in the clinic or emergency room) hemodialysis or intravenous chemotherapy during the past 30 days; hospitalization for at least 1 day during the past 90 days; home intravenous therapy or wound care during the past 30 days; or residence in a long-term care facility.

In addition, $\mathrm{HA}$ infection was defined as an infection that occurred more than 48 hours after admission to the hospital.

Identification and Antimicrobial Susceptibility Testing Isolates of gram-negative bacteria were identified by standard microbiologic methods in the microbiology laboratory using an automated identification system (Vitek System; bioMerieux, Marcy l'Etoile, France). Susceptibilities to antimicrobial agents were determined by the use of an automated susceptibility testing system (Vitek System; bioMerieux). Extended spectrum $\beta$-lactamase (ESBL) production was confirmed by double disk synergy testing, in accordance with the Clinical and Laboratory Standards Institute (CLSI) standards . ${ }^{15}$

\section{Results}

Ninety-two children were diagnosed with gram-negative bacteremia, 64 (71.1\%) with HA bacteremia, 20 (21.1\%) with CA bacteremia, and 8 (7.8\%) with HCA bacteremia. The most recorded criteria found in HA bacteremia were outpatient treatment in the past 30 days, intravascular chemotherapy in the past 30 days, and hospitalization for $>1$ day in the past 90 days. Most of the patients' age was less than 1year (-Table 1). There was no gender difference, and for the underlying comorbidities, there were 12 cases of renal disorder, 12 cases of gastrointestinal disorder, 8 cases of oncology diseases, 6 cases of respiratory disorder, and 13 patients with premature immune deficiency, and there were 3 patients with endocrine disorder ( - Table 2 ) Fever was significantly more common in HA type. Urinary tract infection constituted the major clinical presentation for CA and HCA bacteremia. On the other hand, septic shock/hypotension was the main clinical

Table 1 Age range of pediatric patients with gram-negative bacteremia

\begin{tabular}{|l|l|l|l|l|}
\hline Age range $(y)$ & Community acquired & Health care associated & Hospital acquired & $p$-Value \\
\hline$<1$ (No. 26$)$ & $4(15.4 \%)$ & $1(3.8 \%)$ & $21(80.8 \%)$ & 0.1770 \\
\hline $1-3($ No. 33$)$ & $10(30.3 \%)$ & $3(9.1 \%)$ & $20(60.6 \%)$ & 0.9870 \\
\hline $3-6$ (No. 16$)$ & $3(18 \%)$ & $2(12.5 \%)$ & $11(68.6 \%)$ & 0.2774 \\
\hline $6-12$ (No. 15$)$ & $3(20 \%)$ & $1(6.7 \%)$ & $11(73.3 \%)$ & 0.2150 \\
\hline $12-14$ (No. 2$)$ & $1(6.6 \%)$ & $1(6.7 \%)$ & $0(0 \%)$ & 0.1 \\
\hline Total (No. 92$)$ & $20(21.1 \%)$ & $8(7.8 \%)$ & $64(71.1 \%)$ & 0.7801 \\
\hline
\end{tabular}


Table 2 Comorbidities and clinical characteristics of 92 pediatric patients with community-acquired and health-associated and hospital-acquired gram-negative bacteremia

\begin{tabular}{|c|c|c|c|c|}
\hline Characteristics & $\begin{array}{l}\text { Community acquired } \\
(n=20)\end{array}$ & $\begin{array}{l}\text { Health associated } \\
(n=8)\end{array}$ & $\begin{array}{l}\text { Hospital acquired } \\
(n=64)\end{array}$ & $p$-Value \\
\hline \multicolumn{5}{|l|}{ Underlying disease } \\
\hline Hematological/malignancy ${ }^{8}$ & $4(25 \%)$ & $4(25 \%)$ & $4(50 \%)$ & 0.4795 \\
\hline Renal disease $^{12}$ & $4(17.5 \%)$ & $4(17.5 \%)$ & $8(67 \%)$ & 0.6162 \\
\hline Gastroenterology ${ }^{12}$ & $8(67 \%)$ & $0(0 \%)$ & $4(33 \%)$ & 0.6162 \\
\hline Respiratory $^{6}$ & $2(33 \%)$ & $0(0 \%)$ & $4(67 \%)$ & 0.9352 \\
\hline Endocrine $^{3}$ & $0(0 \%)$ & $0(0 \%)$ & $3(100 \%)$ & \\
\hline Immune deficiency $^{1}$ & $0(0 \%)$ & $0(0 \%)$ & $1(100 \%)$ & \\
\hline Prematurity $^{13}$ & $0(0 \%)$ & $0(0 \%)$ & $13(100 \%)$ & \\
\hline \multicolumn{5}{|l|}{ Clinical presentation } \\
\hline Fever $^{54}$ & $18(22 \%)$ & $6(11 \%)$ & $36(67 \%)$ & 0.0375 \\
\hline Septic shock/hypotension ${ }^{26}$ & $2(8 \%)$ & $0(0 \%)$ & $24(92 \%)$ & 0.0279 \\
\hline Urinary tract infection ${ }^{12}$ & $10(50 \%)$ & $4(33 \%)$ & $2(17 \%)$ & 0.3006 \\
\hline
\end{tabular}

presentation in HA bacteremia indicating that the gramnegative bacteremia is clinically significant. The most common gram-negative bacteria causing bacteremia was Klebsiella pneumoniae (29.3\%), which was isolated in cases with HA bacteremia. This was followed by Escherichia coli (isolated in two types: HA and CA bacteremia, - Table 3). In CA type bacteremia, $25 \%$ of the isolated organisms were E. coli. The antimicrobial susceptibility of the isolated organisms of $\mathrm{CA} / \mathrm{HCA}$, and HA bacteremia showed that majority of the organisms were non-ESBL in $90 \%$, and $10 \%$ of the isolates were ESBL. Three isolates were multi-resistant organism
(MRO) among HA bacteremia ( - Table 4). In comparing the difference between ESBL and non-ESBL isolate among the three types of bacteremia, there was no significant difference in the percentage of ESBL and non-ESBL in CA and HA bacteremia $(p=0.928)$. However, there was a significant difference in the total frequency of isolates between CA and HA bacteremia, regardless whether they were in the ESBL or non-ESBL groups ( $p<0.001,-$ Table 5$)$. However, there was a high mortality rate constituting 17 mortality cases (18.7\%), 14 cases for HA bacteremia and 3 for CA bacteremia (-Table 6).

Table 3 Classification of 92 patients with gram-negative bacteremia among community-acquired, healthcare-associated, and hospital-acquired bacteremia

\begin{tabular}{|l|l|l|l|l|}
\hline Organism & $\begin{array}{l}\text { Community acquired } \\
\boldsymbol{n}=\mathbf{2 0}(\%)\end{array}$ & $\begin{array}{l}\text { Healthcare associated } \\
\boldsymbol{n}=\mathbf{8}(\%)\end{array}$ & $\begin{array}{l}\text { Hospital acquired } \\
\boldsymbol{n}=\mathbf{6 4}(\%)\end{array}$ & $\begin{array}{l}\text { Total } \\
\boldsymbol{n}=\mathbf{9 2}(\%)\end{array}$ \\
\hline Acinetobacter baumannii & $1(5)$ & $2(25)$ & $2(3.1)$ & $5(5.4)$ \\
\hline Acinetobacter Iwoffii & $0(0)$ & $1(12.5)$ & $0(0)$ & $1(1.1)$ \\
\hline Brucella & $2(10)$ & $0(0)$ & $0(0)$ & $2(2.2)$ \\
\hline Burkholderia cepacia & $0(0)$ & $0(0)$ & $1(1.5)$ & $1(1.1)$ \\
\hline Escherichia coli & $5(25)$ & $0(0)$ & $10(15.6)$ & $15(16.3)$ \\
\hline Enterobacter cloacae & $1(5)$ & $2(25)$ & $4(6.2)$ & $7(7.6)$ \\
\hline Haemophilus influenzae & $3(15)$ & $0(0)$ & $0(0)$ & $3(3.3)$ \\
\hline Klebsiella pneumoniae & $0(0)$ & $0(0)$ & $27(42.1)$ & $27(29.3)$ \\
\hline Pseudomonas aeruginosa & $0(0)$ & $2(25)$ & $12(18.7)$ & $14(15.2)$ \\
\hline Pseudomonas flourescens & $0(0)$ & $0(0)$ & $1(1.5)$ & $1(1.1)$ \\
\hline Salmonella species & $6(30)$ & $2(25)$ & $0(0)$ & $8(8.6)$ \\
\hline Salmonella typhi & $1(10)$ & $0(0)$ & $0(0)$ & $1(1.1)$ \\
\hline Serratia marcescens & $0(0)$ & $0(0)$ & $5(7.8)$ & $5(5.4)$ \\
\hline Stenotrophomonas maltophilia & $0(0)$ & $0(0)$ & $2(3.1)$ & $2(2.2)$ \\
\hline Total & 20 & 8 & 64 & $92(100 \%)$ \\
\hline
\end{tabular}


Table 4 The antimicrobial susceptibility of the isolated organisms among community-acquired/health-associated, and hospitalacquired bacteremia $(n=92)$

\begin{tabular}{|l|l|l|l|l|}
\hline Antibiotics & $\begin{array}{l}\text { Resistance } \\
\boldsymbol{n}=\mathbf{9 2}(\mathbf{\%})\end{array}$ & $\begin{array}{l}\text { Community acquired and } \\
\text { health associated } \\
\boldsymbol{n}=\mathbf{2 8}(\%)\end{array}$ & $\begin{array}{l}\text { Hospital acquired } \\
\boldsymbol{n}=\mathbf{6 4}(\%)\end{array}$ & $\mathbf{p}$-Value \\
\hline Pipracillin/tazobactam TAZ & $5(5.5)$ & $0(0)$ & $5(7.8)$ & 0 \\
\hline Bactrim/cotrimoxazole SXT & $18(19.8)$ & $5(18.5)$ & $13(20.3)$ & 0.5657 \\
\hline Meropenem MEP & $5(5.5)$ & $2(7.41)$ & $3(4.7)$ & 0.0674 \\
\hline Imipenem IMP & $6(6.6)$ & $5(18.5)$ & $1(1.6)$ & 0.2793 \\
\hline Gentamicin GM & $14(15.4)$ & $10(37)$ & $4(6.3)$ & 0.6201 \\
\hline Amikacin AK & $4(4.4)$ & $2(7.4)$ & $2(3.1)$ & 0.0405 \\
\hline Augmentin AMC & $16(17.6)$ & $4(14.8)$ & $12(18.8)$ & 0.5663 \\
\hline Ampicillin/Ampiclox AMP & $48(52.7)$ & $6(22.2)$ & $42(65.6)$ & 0.1128 \\
\hline Cephradine CRD & $14(15.4)$ & $4(14.8)$ & $10(15.6)$ & 0.4338 \\
\hline Ciprofloxacin CIP & $11(12.1)$ & $4(14.8)$ & $7(10.9)$ & 0.4447 \\
\hline Aztreonam ATM & $4(4.4)$ & $2(7.41)$ & $2(3.1)$ & 0.0406 \\
\hline Cefoxitin FOX & $13(14.3)$ & $3(11.1)$ & $10(15.6)$ & 0.4597 \\
\hline Cefuroxime/Zinacef CXM & $12(13.2)$ & $0(0)$ & $12(18.8)$ & 0 \\
\hline Cephaloridine CPL & $4(4.4)$ & $0(0)$ & $4(6.3)$ & 0 \\
\hline Chloramphenicol CHL & $2(2.2)$ & $0(0)$ & $2(3.1)$ & 0 \\
\hline
\end{tabular}

Table 5 Difference between ESBL and non-ESBL isolate among community-acquired, healthcare-associated, and hospital-acquired bacteremia

\begin{tabular}{|l|l|l|l|l|}
\hline Organism & $\begin{array}{l}\text { Community acquired } \\
(\boldsymbol{n}=13) \text { and healthcare } \\
\text { associated }(\boldsymbol{n}=7)\end{array}$ & $\begin{array}{l}\text { Hospital } \\
\text { acquired }(\boldsymbol{n}=46)\end{array}$ & Total $(\boldsymbol{n}=66)$ & $p$-Value \\
\hline ESBL & $5(25.0 \%)$ & $11(23.9 \%)$ & $16(24.2 \%)$ & 0.928 \\
\hline non-ESBL & $15(75.0 \%)$ & $35(76.1 \%)$ & $50(75.8 \%)$ & $<0.001$ \\
\hline Total & $20(30.3 \%)$ & $46(69.7 \%)$ & $66(100 \%)$ & \\
\hline
\end{tabular}

Abbreviations: ESBL, extended spectrum $\beta$-lactamase; non-ESBL, nonextended spectrum $\beta$-lactamase.

Note: There is no significant difference in the percentage of ESBL and non-ESBL in community-acquired and hospital-acquired bacteremia $(p=0.928)$.However, there is a significant difference in the total frequency of isolates between community-acquired and hospital-acquired bacteremia, regardless of ESBL or non-ESBL $(p<0.001)$.

\section{Discussion}

Recent data exist on the differentiation of HCA bacteremia from HA bacteremia and CA bacteremia. ${ }^{16}$ In this study, comparing the epidemiology of CA, HCA, and HA bloodstream infections, it was found that health-associated bacteremia (7.8\%) was of low incidence unlike other studies in which there were higher incidences of HCA bacteremia. This is similar to a study performed in Spain that found that $18 \%$ were CA, 24\% were HCA, and 58\% were $\mathrm{HA}^{17}$ Hoenigl et al found that of the total 1,143 patients diagnosed with bacteremia, HCA accounted for $63.7 \%$ and CA for $36.3 \%$ cases. $^{18}$ Similarly, Friedman's group reported that $50.9 \%$ of their 159 cases of gram-negative bacteremia were HCA. ${ }^{9}$ Unlike this study, in the study by Hoenigl et al, the study cohort had slightly more male subjects ( 56.1 vs. $50.2 \%, p=0.044$ ) than females. ${ }^{18}$ In this study, analyzing the causative pathogens, we found that the most common gram-negative bacteria

Table 6 Difference between the mortality rate in hospital-acquired and community-acquired bacteremia

\begin{tabular}{|l|l|l|l|}
\hline Hospital acquired $(n=64)$ & Community acquired $(n=20)$ & Total & $p$-Value \\
\hline $14(82.4 \%)$ & $3(17.6 \%)$ & 17 & 0.1228 \\
\hline
\end{tabular}


causing bacteremia was K. pneumoniae and was found to be significantly associated with the HA bacteremia. The causative pathogens were comparable between patients with $C A$ and those with HCA bacteremia. In a recent prospective cohort study, in 672 patients enrolled with positive peripheral blood cultures (192 CA, 85 HCA, and 395 HA), E. coli was found to be the most frequently isolated pathogens. Escherichia coli was isolated more frequently in patients with community-onset bacteremia, and Pseudomonas species were isolated more frequently among those with HA bacteremia. ${ }^{18}$ In agreement with previous reports, the most frequently seen underlying diseases in patients with gramnegative bacteremia were malignancies. ${ }^{19}$ Compared with patients with HCA bacteremia and CA bacteremia for patients with HA bacteremia, the clinical presentation of urinary tract infection was higher in HCA bacteremia and CA bacteremia-4 (50\%) and 10 (50\%), respectively, versus 2 (1.3\%) for HA bacteremia. Marschall et al reported contradicting results with findings of 38 (28.8\%) versus 13 (14.4\%) cases of bacteremia for the urinary tract when compared with HA bacteremia and HCA bacteremia. ${ }^{20}$ This finding is in accordance with the previous study that reported that HCA bacteremia was associated with increased 30- and 90-day mortality rates when compared with CA bacteremia. ${ }^{21}$

In other studies, the mortality rate of HCA infection seemed to be generally higher than that of CA infection and was similar to that of hospital infection. ${ }^{10}$

In this study, all the isolates of HCA were multisensitive, contrary to a study from Korea in which a total of 240 patients were infected with community-onset K. pneumoniae bacteremia, 140 (58.3\%) were defined as HCA infection cases, and the remaining 100 patients were classified as CA infections. Patients infected with HCA bacteremia showed significantly different clinical and microbiological characteristics compared with those infected with CA bacteremia. HCA K. pneumoniae bacteremia was characterized by more antibiotic-resistant pathogens (ciprofloxacin resistance, 12.9 [18/140] vs. 4.0\% [4/100], $p=0.02$ ) and ESBL production (12.1 [17/140] vs. $4.0 \%$ [4/100], $p=0.03$ ) than CA bacteremia. 22

A recent study from Saudi Arabia reported their experience of HA catheter-related blood stream infections (CRBSIs). CRBSI resulted in 60 admissions with a median of 182 days of hospital stay and 74 changes of central venous catheters. The rate of CRBSI was 2.9 per 1,000 catheter days. Staphylococcus species were the most prevalent pathogens (32\%), followed by $K$. pneumoniae (5\%). ${ }^{23}$

\section{Conclusion}

This study concluded that the epidemiology, causative pathogens, and mortality differed markedly between community-onset and HA bacteremia, while differences between CA and HCA bacteremia were by far less pronounced. HA bacteremia was associated with an increase in the risk of mortality when compared with CA bacteremia.

Our study had some limitations. One is the small sample size, a larger study should be performed to validate the findings of this study. Future studies should focus on recog- nizing CA and HCA bacteremia due to resistant organisms. Nonetheless, to the best of our knowledge, this is the first study comparing the clinical and bacteriological characteristics of HCA, HA, and CA gram-negative bacteremia in Saudi pediatric patients.

\section{Conflict of Interest}

None.

\section{Funding}

This work was financially supported by Prince Abdullah bin Khaled Celiac Disease Research Chair, Vice Deanship of Research Chairs, King Saud University, Riyadh, Saudi Arabia.

\section{References}

1 Laupland KB. Defining the epidemiology of bloodstream infections: the 'gold standard' of population-based assessment. Epidemiol Infect 2013;141(10):2149-2157

2 Henderson KL, Müller-Pebody B, Johnson AP, Wade A, Sharland M, Gilbert R. Community-acquired, healthcare-associated and hospital-acquired bloodstream infection definitions in children: a systematic review demonstrating inconsistent criteria. J Hosp Infect 2013;85(02):94-105

3 Sick AC, Tschudin-Sutter S, Turnbul AE, Weissman SJ. Tamma. Empiric combination therapy for gram-negative bacteremia. Pedia 2014;133(05):1148-1155

4 Pedersen G, Schønheyder HC, Sørensen HT. Source of infection and other factors associated with case fatality in communityacquired bacteremia-a Danish population-based cohort study from 1992 to 1997. Clin Microbiol Infect 2003;9(08):793-802

5 Søgaard M, Nørgaard M, Dethlefsen C, Schønheyder HC. Temporal changes in the incidence and 30-day mortality associated with bacteremia in hospitalized patients from 1992 through 2006: a population-based cohort study. Clin Infect Dis 2011;52(01):61-69

6 Siegman-Igra Y, Fourer B, Orni-Wasserlauf R, et al. Reappraisal of community-acquired bacteremia: a proposal of a new classification for the spectrum of acquisition of bacteremia. Clin Infect Dis 2002;34(11):1431-1439

7 Friedman ND, Kaye KS, Stout JE, et al. Health care-associated bloodstream infections in adults: a reason to change the accepted definition of community-acquired infections. Ann Intern Med 2002;137(10):791-797

8 De Bus L, Coessens G, Boelens J, Claeys G, Decruyenaere J, Depuydt P. Microbial etiology and antimicrobial resistance in healthcareassociated versus community-acquired and hospital-acquired bloodstream infection in a tertiary care hospital. Diagn Microbiol Infect Dis 2013;77(04):341-345

9 McDonald JR, Friedman ND, Stout JE, Sexton DJ, Kaye KS. Risk factors for ineffective therapy in patients with bloodstream infection. Arch Intern Med 2005;165(03):308-313

10 Shorr AF, Tabak YP, Killian AD, Gupta V, Liu LZ, Kollef MH. Healthcare-associated bloodstream infection: adistinct entity? Insights from a large U.S. database. Crit Care Med 2006;34(10): 2588-2595

11 Vallés J, Calbo E, Anoro E, et al. Bloodstream infections in adults: importance of healthcare-associated infections. J Infect 2008;56 (01):27-34

12 Tiwari DK, Golia S, K TS, C LV, A study on the bacteriological profile and antibiogram of bacteremia in children below 10 years in a tertiary care hospital in Bangalore, India. J Clin Diagn Res 2013;7 (12):2732-2735

13 Sharma M, Yadav A, Goel N, Chaudary U. Microbial profile of septicemia in children. Ind J for the Practicing Doctor 2013;5(04):9-10 
14 Lim CJ, Cheng AC, Kong DC, Peleg AY. Community-onset bloodstream infection with multidrug-resistant organisms: a matched case-control study. BMC Infect Dis 2014;14:126

15 Clinical and Laboratory Standards Institute. Performance standards for antimicrobial susceptibility testing; 22nd Informational Supplement (Vol 32) Clinical and Laboratory Standards Institute; PA, USA: 2012

16 Rodríguez-Baño J, López-Prieto MD, Portillo MM, et al; SAEI/ SAMPAC Bacteraemia Group. Epidemiology and clinical features of community-acquired, healthcare-associated and nosocomial bloodstream infections in tertiary-care and community hospitals. Clin Microbiol Infect 2010;16(09):1408-1413

17 Kollef MH, Zilberberg MD, Shorr AF, et al. Epidemiology, microbiology and outcomes of healthcare-associated and communityacquired bacteremia: a multicenter cohort study. J Infect 2011;62 (02):130-135

18 Hoenigl M, Wagner J, Raggam RB, et al. Characteristics of hospitalacquired and community-onset blood stream infections, South-east Austria. PLoS ONE 2014;8(09):e104702www.plosone.org
19 Chang TY, Lee CH, Liu JW. Clinical characteristics and risk factors for fatality in patients with bloodstream infections caused by glucose non-fermenting gram-negative Bacilli. J Microbiol Immunol Infect 2010;43(03):233-239

20 Marschall J, Fraser VJ, Doherty J, Warren DK. Between community and hospital: healthcare-associated gram-negative bacteremia among hospitalized patients. Infect Control Hosp Epidemiol 2009;30(11):1050-1056

21 Rodriguez-Bano J, Picon E, Gijon P, et al. Risk factors and prognosis of nosocomial bloodstream infections caused by extended-spectrum-beta-lactamase-producing Escherichia coli. J Clin Microbiol 2010;48(05):1726-1731

22 Lee JA, Kang CI, Joo EJ, et al. Clinical and microbiological characteristics of healthcare-associated infections in community-onset Klebsiella pneumoniae bacteremia. Infect Chemother 2012;44(02):56-61

23 Al-Tawil ES, Almuhareb AM, Amin HM. Catheter-related blood stream infection in patients receiving long-term home parenteral nutrition: tertiary care hospital experience in Saudi Arabia. Saudi J Gastroenterol 2016;22(04):304-308 